\title{
Builiding Methods for a Proactive Prescription Drug Surveillance System
}

\author{
Fan Xiong*1,2 \\ ${ }^{1}$ CDC / CSTE Applied Epidemiology Fellowship Program Class of XIII, Atlanta, GA, USA; ${ }^{2}$ Kansas Department of Health and \\ Enviroment - Topeka, KS, Topeka, KS, USA
}

\section{Objective}

This study aims to show the application of longitudinal statistical and epidemiological methods for building a proactive prescription drug surveillance system for public health.

\section{Introduction}

Prescription Drug Monitoring Programs (PDMPs) are operating in 49 states and several U.S. territories. Current methods for surveillance of prescription drug related behaviors, include the mean daily dosage of morphine milligram equivalent (MME) per patient, annual percentage of days with overlapping prescriptions per patient, and annual multiple provider episodes for multiple controlled substance prescription drugs per patient that are described elsewhere. ${ }^{1,2}$ This work builds on these efforts by extending longitudinal methods to prescription drug behavior surveillance in order to predict risks associated with prescription drug use.

\section{Methods}

Schedule II prescription opioids from January 1, 2014 to February 29, 2016 from the Kansas Tracking and Reporting of Controlled Substances (KTRACS) was used for this analysis. Prescription opioids were linked to the 2016 version of the morphine milligram equivalent conversion table from the National Center for Injury Prevention and Control. ${ }^{3}$ Population estimates were based on the 2015 County Vintage single-year of age bridged-race estimates from the National Center for Health Statistics and used to calculate age-adjusted rates. A daily high dose opioid prescription was defined as having greater than or equal to 90 morphine milligram equivalent. Since this is a unit-day measure with patients experiencing multiple daily high dose opioid days, the Prentice, William, and Peterson (PWP) recurrent event model was used to estimate the number of high-dose opioid days for Kansas patients by gender and age groups. ${ }^{4,5}$ Start time was the first prescription date with a high-dose opioid and stop time was the next high-dose opioid date during a study period from January 1, 2014 to Feb 29, 2016. The PWP model is a statistical model that allows for the estimation of covariates on an event history (i.e. total time with prescription opioids, specifically high-dose opioids). Analysis was completed with a stratified Cox-proportional hazard model, sandwich covariance for dependent observations, and statistical significance was assessed with a Wald Chi-square. PROC PHREG in SAS/STAT(R) 14.1 was used since it has a new FAST option for fitting large proportional counting process hazard model.

\section{Results}

The age-adjusted rate of daily high-dose opioid patients was 3.2 patients per 100 Kansas population-year (95\% CI: $3.1-3.2$ ). Kansas patients aged 85 and older had the highest age-specific rate of 11.7 (95\% CI: $11.5-11.9)$. Preliminary recurrent event analysis shows on average nearly a quarter of approximately 50 million Schedule II opioid patient days were high-dose opioid patient days among 785,514 Kansan patients with any prescribed opioid history. In an initial result stratified by the number of high-dose opioid days and adjusting only for age, males on average had approximately $7 \%$ higher hazard of recurrent Schedule II high-dose opioid prescription days than females $(\beta$ : 0.07 , S.E: $0.002, \mathrm{p}<0.0001)$. Kansas patients aged 45 to 54 compared to Kansas patients 85 and older on average had approximately $14 \%$ higher hazard of recurrent Schedule II highdose opioid prescription days ( $\beta$ : 0.14, S.E: $0.007, \mathrm{p}<0.0001)$.

\section{Conclusions}

This work demonstrates the application of survival analysis techniques to estimate the population at risk for high-dose opioids, which varies by the length of the total opioid prescription history. Early results from the recurrent event analysis showed that Kansas male and patients aged 45 to 54 years had the longest history of high-dose opioids. Annual cross-sectional population estimates may incorrectly estimate the estimated risk of high-dose prescription opioids since it assumes all patients have the same prescription history. PDMPs are longitudinal databases. Survival analysis methods like recurrent event models can leverage the longitudinal structure to more precisely estimate risk statistics. Future work includes incorporation of health outcomes data and further prescription covariates to assess the timing and intensity of opioid potency escalation.

\section{Keywords}

Prescription opioids; morphine milligram equivalent; prescription drugs

\section{Acknowledgments}

This work was supported in part by an appointment to the Applied Epidemiology Fellowship Program administered by the Council of State and Territorial Epidemiologists (CSTE) and funded by the Centers for Disease Control and Prevention (CDC) Cooperative Agreement Number 1U38OT000143-03. Dr. Belle Federman at the Kansas Department of Health and Environment for best methods to communicate the findings of this study.

\section{References}

1. Paulozzi LJ, Strickler GK, Kreiner PW, Koris CM. Controlled substance prescribing patterns - prescription behavior surveillance system, eight states. 2013. MMWR Surveill Summ. 2015 Oct 16;64(9):1-4.

2. Definitions of PBSS Measures. Brandeis' University Center of Excellence for Prescription Drug Monitoring Program's Prescription Behavior Surveillance System. Available at http://www. pdmpexcellence.org/sites/all/pdfs/Definitions $\% 20$ of $\% 20$ PBSS $\% 20$ Measures.pdf

3. National Center for Injury Prevention and Control. CDC compilation of opioid analgesic formulations with morphine milligram equivalent conversion factors, 2016 version. Atlanta, GA: Centers for Disease Control and Prevention. 2016. Available at http://www.pdmpassist. org/pdf/BJA_performance_measure_aid_MME_conversion.pdf.

4. Prentice, R. L., Williams, B. J., and Peterson, A. V. (1981), "On the Regression Analysis of Multivariate Failure Time Data," Biometrika, 68. 373-379.

5. Amorim LD, Cai J. Modelling recurrent events: a tutorial for analysis in epidemiology. International journal of epidemiology. 2015 Feb 1. 44(1):324-33.

\section{${ }^{*}$ Fan Xiong}

E-mail: fxiong@kdheks.gov 